\title{
Possible names: The role of syntax-semantics mappings in the acquisition of nominals*
}

\author{
Paul Bloom \\ Department of Psychology, University of Arizona, Tucson, AZ 8572I, USA
}

Many scholars have posited constraints on how children construe the meanings of new words. These include the restriction that new words refer to kinds of whole objects (Markman and Hutchinson 1984), that words describing solid objects refer to individuated objects while words describing non-solid substances refer to portions of substance (Soja et al. 1991), and that count nouns that name objects are generalized on the basis of shape (Landau et al. 1988). There are theoretical and empirical problems with these proposals, however. Most importantly, they fail to explain the fact that children rapidly acquire words that violate these constraints, such as pronouns and proper names, names for substances, and names for non-material entities. The theory defended here is that children and adults possess mappings from grammatical categories ('count noun', 'mass noun', and 'noun phrase') to abstract semantic categories; these mappings serve to constrain inferences about word meaning. Evidence from developmental psychology and linguistic theory is presented that suggests that even very young children possess such mappings and use them in the course of lexical development. Further issues - such as the possibility of developmental change, the precise nature of these semantic categories, and how children learn words prior to the acquisition of syntax - are also discussed. It is concluded that although these syntax-semantics mappings are not by themselves sufficient to explain children's success at word learning, they play a crucial role in lexical development. As such, only a theory that posits a deep relationship between syntax and semantics can explain the acquisition and representation of word meanings.

'Language ... must have its perfectly exclusive pigeon-holes and will tolerate no flying vagrants. Any concept that asks for expression must submit to the classificatory rules of the game ... It is almost as though at some period in the past

* I am grateful to Felice Bedford, Susan Carey, Frank Keil, Ellen Markman, Janet Nicol, Mary Peterson, Nancy Soja, an anonymous reviewer, and especially Karen Wynn for their very helpful comments on earlier versions of this paper. This work was supported by a NIH Biomedical Research Support Grant. 
the unconscious mind of the race had made a hasty inventory of experience, committed itself to a premature classification that allowed of no revision, and saddled the inheritors of its language with a science that they no longer quite believed in nor had the strength to overthrow. Dogma, rigidly prescribed by tradition, stiffens into formalism. Linguistic categories make up a system of surviving dogma - dogma of the unconscious.'

Edward Sapir, Language (1921: 99-100)

\section{Introduction}

One of the deepest mysteries in the study of language development is how children learn the meanings of words. A child's vocabulary grows at an extraordinary rate - one estimate is that children acquire about nine new words a day from the age of 18 months to six years (Carey 1978) - and there is little understanding of how this process takes place. This ignorance is due at least in part to the fact that there is no consensus on what it is for someone to possess 'the meaning of a word' (for discussion, see Carey 1982, Lakoff 1987, Premack 1990). In general, no theory of acquisition can be complete without some understanding of the nature of what must be acquired.

A further difficulty concerns the nature of the learning problem itself. Word learning is standardly viewed as an inductive process and, as Goodman (1983) has stressed, there is an infinity of logically possible generalizations that one can make on the basis of a finite set of instances. To take a specific example, consider an adult pointing to Fido and saying to a child 'Look at the dog'. Imagine that somehow the child is capable of determining what the word is intended to describe, i.e., it describes Fido, not the child, or the finger, or the act of pointing, etc. Imagine further that the child can segment the utterance into words and can determine that the relevant word is 'dog', not 'look', 'at', or 'the'. Still, there are countless possible meanings of this novel word. It could refer to the basic-level kind (dogs), but it could also refer to a subordinate kind (poodle), or a superordinate kind (animal), or to the individual (Fido). It could refer to the color of the entity being pointed to (brown), to its shape (oblong), or its size (large). It could refer to a part of the dog (tail). It could refer to the front half of the dog, to dogs until the year 2000 and then to pigs, to all dogs and all pencils, to all dogs and also to Richard Nixon. To modify an example from Quine (1960), it could even refer to undetached dog parts. 
From a logical standpoint, all of these examples are possible, since they are all consistent with the ostensive act. From a psychological standpoint, however, some of these possibilities are ludicrous - no child would ever construe the word 'dog' as referring to just the front half of Fido, or to the category of 'dogs and pencils'. Any theory of word learning must explain why some word meanings are more natural than others, and how children determine which of the set of natural meanings that a word could have actually corresponds to its meaning in a given language.

More generally, any succesful inductive procedure requires that hypotheses be somehow ordered or ranked (Fodor 1975, Goodman 1983) and one possibility is that there exist constraints that rule out (or bias against) entire classes of hypotheses. In the domain of language development, Markman and her colleagues have presented the following two constraints, which are argued to be special to the domain of word learning (e.g., Markman and Hutchinson 1984, see Markman 1990 for a review):

\section{Whole Object constraint:}

'... a novel label is likely to refer to the whole object and not to its parts, substance, or other properties.' (Markman 1990: 59)

\section{Taxonomic constraint:}

'... labels refer to objects of the same kind rather than to objects that are thematically related' (Markman 1990: 59). Thematically related entities include those that fall into 'spatial, causal, temporal, or other relations' such as a dog and its bone, a dog and the tree that it is under, a dog and the person who is petting it, and so on. Although children are sensitive to these sorts of relations in non-linguistic tasks (for instance, they will put a dog and a bone together when asked to sort objects into different piles), this constraint forces them to attend to taxonomies (such as the kind 'dog') when faced with the task of inferring the meaning of a new word.

Another proposal, advanced by Soja et al. (1991: 182-183), is that the following two procedures apply in the process of word learning:

\section{Procedure 1:}

Step 1: Test to see if the speaker could be talking about a solid object; if yes,

Step 2: Conclude that the word refers to individual whole objects of the same type as the referent. 
Procedure 2:

Step 1: Test to see if the speaker could be talking about a non-solid substance; if yes,

Step 2: Conclude that the word refers to portions of substance of the same type as the referent.

Finally, Landau et al. (1988, 1992) posit the following bias in lexical acquisition:

\section{Shape bias:}

'... the bias to group objects by shape in the presence of a novel count noun

...' (Landau et al. 1992: 87)

There is by now a large body of evidence showing that 2- and 3-year-olds behave in accordance with these posited constraints. When taught a word for a novel object, children tend to categorize the word as referring to other whole objects of the same kind; they will not extend the word to entities sharing a 'thematic' relation (Markman and Hutchinson 1984, Waxman and Gelman 1986) and will not initially interpret it as referring to a part of the object, a property of the object, or the stuff that the object is made of (Baldwin 1989, Clark 1973, Macnamara 1982, Markman and Wachtel 1988, Soja et al. 1991, Taylor and Gelman 1988). In contrast, when taught a word for a novel non-solid substance, they will tend to generalize the word on the basis of the kind of substance (perhaps using texture and color as cues), and ignore properties such as shape and size (Soja 1987, 1992; Soja et al. 1991). Finally, when taught count nouns that describe objects, children will tend to generalize these nouns on the basis of shape, not color, size, or texture (Baldwin 1989, Landau et al. 1988).

Nevertheless, there are reasons to doubt that these precise constraints are present in the minds of young children. For one thing, they are false of adult language - all languages have words that do not refer to taxonomies, words that do not refer to whole objects, and count nouns that name kinds of objects that do not share a common shape. Below it is argued that these counterexamples are also present in the language of very young children. A further concern is that the sole motivation for positing these constraints is their role in solving the word learning problem. It would be preferable to derive these constraints from deeper properties of language and cognition, instead of having to simply stipulate them.

In this paper, I present a theory of where constraints on word meaning 
come from. It is often maintained that children possess mappings between syntax and semantics which facilitate grammatical and lexical development (e.g., Bloom 1990a,b; 1994a,b; Brown 1957, Carey 1982, Gleitman 1990, Grimshaw 1981, Katz et al. 1974, Landau and Gleitman 1985, Landau et al. 1988, 1992; Macnamara 1982, 1986; Macnamara and Reyes 1990, Naigles 1990, Pinker 1984, 1989; Taylor and Gelman 1988, Waxman 1990). It is argued here that the existence of certain syntax-semantics mappings in the domain of nominals makes it unnecessary to posit special word-learning constraints and that a theory based on such mappings allows for a better explanation of how young children learn words. ${ }^{1}$

\section{Problems with proposed constraints}

\subsection{Description of adult language}

As Markman (1990) notes, the whole object constraint is false at least for the lexicons of older children and adults. There exist words that refer to properties ('happy'), to actions ('hit'), to spatial relations ('under'), to substances ('water') and so on. In general, adjectives, verbs, prepositions, and mass nouns do not refer to whole objects.

One apparent solution would be to redescribe the constraint as only applying to count nouns, and thus exclude examples from other grammatical categories. But the generalization still does not hold. Some count nouns describe whole objects ('dog'), but most do not. Within the domain of count nouns that describe material entities, some refer to collections of objects ('forest', 'bikini', 'flock'), while others refer to parts of objects ('finger', 'foot',

1 Other constraints on word learning that have been proposed include mutual exclusivity, which is that words cannot have overlapping extensions (Markman and Wachtel 1988), and the principle of contrast (Clark 1987), which states that there are no synonymous forms in language. As with the constraints discussed above, mutual exclusivity is clearly false of adult language (as it excludes pairs of words such as 'dog' and 'animal') and there is considerable debate over how well it applies to the language of young children (see Au and Glusman 1990, Gathercole 1987, Mervis et al. 1991, Nelson 1988). The principle of contrast may be correct for adult language but it is not by itself sufficient to constrain the child's inferences and must work in accord with other principles. Neither of these constraints can be captured in terms of the sorts of syntax-semantics mappings discussed here, but it is possible, as suggested by both Clark (1990) and Gathercole (1987), that these constraints might also reduce to deeper properties of language and cognition. In particular, a prohibition on certain forms of lexical overlap may be derivable from children's knowledge of pragmatic principles, e.g., of Gricean maxims of communication. 
'handle'). (In fact, some nouns refer to parts of objects that can never appear as discrete entities, such as 'surface' or 'coating'.) The largest class of exceptions are count nouns like 'nap', 'idea', 'race', and 'dream', which do not refer to material entities at all.

Given that the shape bias is intended to apply only to count nouns that name objects, words like 'nap' do not violate this bias. But other nouns do, such as names for collections ('army', 'family'), superordinates ('animal', 'weapon') and relationship terms ('brother', 'friend'). In fact, it is far from clear that shape is the crucial dimension even for count nouns that refer to basic-level discrete whole objects, which are those considered by Landau et al. (1988). As Soja et al. (1991, 1992) argue, even for young children, shape is not criterial in determining the extension of count nouns like 'dog' or 'skunk'; something can be thought of as a dog even if it is shaped like a cat (Keil 1989). What Landau et al. have found is that children prefer to generalize novel nouns referring to discrete material objects on the basis of shape as opposed to size or texture. But this is a far weaker conclusion than the claim that count nouns, or even count nouns referring to objects, "correspond to categories whose members have similar shapes' (Landau et al. 1988: 316). On the contrary, there is only a small number of nouns, such as 'square', 'globe', and 'pyramid', where shape is an essential property of how they are used.

The taxonomic constraint is also false for adults. While it is truc that words cannot refer to chains of thematically related categories, there are nominals that do not refer to taxonomies; these include pronouns and proper names ('he', 'Fred', 'Canada'), which refer to particular individuals, and do not generalize to other entities.

Of all of the constraints proposed above, only those advanced by Soja et al. (1991) are largely correct with regard to adult language - but (once again) only when we replace 'word' with 'noun' (as they themselves suggest, p. 203). It is clearly false that any word used to describe a solid object can be extended to objects of the same type, since the word could be describing a property of the object ('red'), the state of the object ('resting'), and so on, and the same observation applies to the claim that any word used to describe a non-solid substance can be extended to that type of substance. But although these procedures work better when their domain is restricted to nouns, there are still cases where they fail. Some mass nouns, like 'wood' and 'metal', describe solid objects but are not extended to objects of the same type; they are extended to objects composed of the same material. The opposite sort of counterexample concerns count nouns like 'pile' and 'puddle'; they describe substances, but are extended on the basis of configuration, not substance-kind. 


\subsection{Description of child language}

The same counterexamples found in adult language also show up in the spontaneous speech of young children. As Nelson $(1988,1990)$ has stressed, although nouns (or nominals) may be the largest single class of words in the beginning vocabulary of some young children (Gentner 1982), it is false that all, or even most, of children's first words are nouns. Children's early lexicons include expressions like 'more', 'bye', 'hit', 'want', 'up', and 'no' and these clearly refute any claim that children's first words must refer to kinds of whole objects (see also Benedict 1979, Gopnik and Choi 1990).

What about the more specific claim that all of the nominals used by young children refer to kinds of whole objects? This still leaves us with constraints that are descriptively inadequate, however. One problem concerns words like 'milk' and 'water', which refer to kinds, but to kinds of substances, not objects. A second problem concerns pronouns and proper names, which do not refer to kinds. Given these counterexamples, one could further revise the constraint proposal as follows:

\section{Constraint hypothesis:}

In the lexicons of young children, count nouns refer to kinds of whole objects

In some discussions of the taxonomic and whole object constraints, this is alluded to as the most descriptively adequate version of the constraint theory and Markman (1989), although usually defining the constraints as applying to 'labels', sometimes describes it in this manner, as limited to count nouns.

This revision still has difficulties, however. Even two-year-olds possess names for collections like 'forest' and 'family', despite the fact that these count nouns violate the whole object constraint (Bloom and Kelemen, under review; Callanan and Markman 1982). Nelson (1990: 335) gives as a further example "the many abstract social and cultural concepts that are incorporated into the language and presented in passing to children, who pick them up seemingly without effort' and she lists some words found in the speech of 20-month-old children; these include count nouns such as: 'bath', 'breakfast', 'friend', 'week', and 'uncle'. Finally, Nelson et al. (1993) analyzed the speech of 4520 -month-olds and found that, although these children possessed more nouns than any other category, only about half of these nouns referred to basic-level classes of objects; many referred to locations ('beach'), temporal entities ('day'), and events ('party'). 
The rapid acquisition of such words is clearly problematic for the whole object constraint and the taxonomic constraint. It also poses a difficulty for the shape bias. As noted by Soja et al. (1991, 1992), it would be surprising indeed if children extended words like 'uncle' and 'clock' on the basis of shape; if they did, they would be unable to use these words in any way similar to adults.

Finally consider the Soja et al. (1991) procedures, which state that words describing objects refer to kinds of individual whole objects and words describing substances refer to portions of substance. Revising their proposal so that it applies only to nouns, this predicts that children should be unable to acquire solid substance names ('wood') and names for bounded substances ('puddle'). But in fact even 2-year-olds can learn solid substances names (Prasada 1993), and there is evidence from Soja (1992) suggesting that they can also acquire names for bounded entities (see section 4.2 for discussion). It is worth noting, however, that these are not children's first guesses as to a word's meaning. For instance, when a word is used to describe a novel bounded entity, children's first interpretation of its meaning is that it refers to the kind of object, not the stuff that the object is made out of, and this holds regardless of the syntax in which the word is presented (Au and Markman 1987, Dickinson 1988, Markman and Wachtel 1988, Soja 1987). Nevertheless, the fact that words like 'wood' are acquired at all militates against the claim that children possess the procedures posited by Soja et al. (1991).

None of the counterexamples discussed above necessarily refutes the hypothesis that these constraints exist. One possibility, discussed in detail by Markman (1989), is that although they are present at the start of lexical development, the constraints can be abandoned or 'overridden' in the course of language development (possibly as the result of the application of other constraints; see footnote 1). More generally, they can be viewed as default conditions which only apply in the absence of certain countervailing circumstances, and such circumstances may be present at any stage of lexical development.

Under this interpretation of what constraints are, however, it is unclear whether any degree of understanding on the part of young children could refute these proposals. For instance, pronouns and proper names are acquired very early, often before children have learned any other noun that describes people (such as 'person', 'man', and 'woman'). As such, constraints such as Mutual Exclusivity cannot block the child from interpreting words like 'Fred' and 'she' incorrectly, as names for kinds of whole objects. In fact, these sorts of crrors do not occur (see Macnamara 1982) - but a reasonable reply by a 
constraint-theorist is that the special status of what pronouns and proper names refer to (i.e., people) causes children to override the taxonomic assumption in such cases. In general, the fact that these constraints are posited as default conditions makes it important to provide some theory of what other rules and constraints can override them. Without such a theory, the constraint proposal runs the risk of begging all of the difficult questions.

In sum, the theories of Markman, Landau et al., and Soja et al. cannot account for most of the words that children acquire. But the very same induction problem that exists for the acquisition of a word such as 'dog' also exists for words such as 'Fred', 'water', and 'forest', and thus the very same arguments for the necessity of constraints also apply. The goal of a theory of lexical development is to account for the acquisition of all words, not just names for kinds of objects, and this motivates an effort to explain the acquisition of object names in the context of a more general theory of word learning.

\subsection{Learning issues}

Where do constraints on word learning come from? In this regard, it is worth echoing Nelson's (1988) complaint that some of the theorists who posit these constraints are vague as to whether or not they are presumed to be unlearned. There is a strong argument, however, that the only claim consistent with the idea of such constraints is that at least some of them are present prior to the onset of word learning. The motivation for positing constraints in the first place is to explain how children solve the induction problem and learn words. From this perspective, it would be contradictory to claim that (for example) children learn that words describe kinds of whole objects, as this would require that they first learn the meanings of some words and then notice that they tend to refer to whole objects. This would imply that children are able to acquire words without this constraint, and thus one could not appeal to its existence as an explanation of how children initially solve the word learning problem.

Consider also the specific proposal that children induce that members of certain grammatical categories tend to share certain meaningful properties, and that this is the origin of some of the constraints. Landau et al. (1988: 317) suggest that 'the development of a same-shape preference in children may originate in language learning, specifically in the process of learning count nouns. Many of the words acquired by early language learners do in 
fact partition the world according to the shapes of the objects in it ... young children very quickly realize this, abstracting a rule from their early word learning experience that says shape is the critical factor in decisions about the extensions of these nouns. Then they use this rule when encountering new nouns and new classes, thus immensely simplifying and speeding up the mapping of the one onto the other'. While possible, this assumes that children are able to learn the correct meanings of count nouns (and can thus infer that they tend to refer to shape) prior to the onset of the shape bias. Thus although this bias might facilitate word learning later on, we are still left with the problem of what constrains children's inferences in the first place.

If the constraints are unlearned, what is their precise nature? Do they constitute a subpart of a distinct language acquisition mechanism that exists solely to facilitate the learning of words? This is certainly conceivable, but it would be preferable to motivate these constraints on word learning in terms of more general properties of children's linguistic and cognitive competence. One specific proposal, advanced below, is that these constraints emerge from other properties of children's knowledge; in particular, from children's grasp of syntax-semantics mappings.

\section{Syntax-semantics mappings as a theory of constraints on word learning}

Below, I discuss certain mappings between syntax and semantics and argue that they effectively subsume the constraints reviewed above. These mappings have two advantages over the constraints: (i) they have independent linguistic and psychological support and (ii) they provide a framework to explain the acquisition of all nominals, including those such as 'dog', 'water', 'wood', 'forest', and 'Fred', and also including those count nouns that are not names for material entities.

\subsection{Lexical categories vs. phrases}

Markman (1990) briefly discusses a theory of the origin of the taxonomic constraint, which is based on Di Sciullo and Williams (1987) and states that 'words are generic in meaning in a way that phrases are not'; words do not make reference to specific things, times, or places. She suggests that the taxonomic constraint may be a consequence of this property of words. This insight seems fundamentally correct, but the explanation needs to be modified 
in order to account for words like 'Fred' and 'she' which refer to individuals and are not 'generic' in the sense discussed by Di Sciullo and Williams.

An alternative is that the relevant distinction is grammatical, and related to how languages use syntactic categories to express meaningful notions and relations (Bloom 1990a, Jackendoff 1983). In particular, it is not words that have generic reference - it is categories such as nouns and verbs. Nouns like 'dog' and 'water' are generic in the sense that they can be extended to an indefinite number of novel instances (an infinity of different dogs, an infinity of different portions of water). Put differently, they refer to kinds, not to particular individuals or entities.

In contrast, noun phrases (NPs) like 'the big dog' can be conceptualized as referring to individuals, and not to kinds. The standard examples of this are when nouns combine with quantifiers to become NPs. Thus 'a dog' can pick out a particular individual that happens to be a dog, 'those big dogs' picks out those dogs that have the property of being big, and so on. (See Parsons, 1990, for a discussion of how a similar analysis can apply to verbs and VPs.)

The distinction between words and nouns is crucial here, since some words are NPs, not nouns. This allows us to explain the peculiar status of pronouns and proper names. From the standpoint of grammar, they are lexical NPs (see Bloom 1990b). With regard to their role in syntactic structure, words like 'Fred' and 'he' behave like phrasal NPs such as 'the dog' and thus cannot appear with adjectives and determiners. Under the hypothesis that pronouns and proper names are NPs, we can posit the following mappings:

Mapping 1: NPs refer to individuals.

Mapping 2: Count nouns and mass nouns refer to kinds.

It is likely, however that Mapping 1 is too strong; there are NPs that appear not to refer at all. In a language like English, these include expletive pronouns, such as 'it' as in 'it is raining' or 'there' as in 'there is trouble brewing'. One theory of why such semantically empty NPs exist is because of a requirement in English that all tensed sentences must have overt subjects (Chomsky 1981), even if these subjects play no meaningful role. In languages like Italian, where overt subjects are not necessary, one can say the equivalent of 'is raining', while in English, it is necessary to add the meaningless NP 'it' in order to satisfy the grammatical requirement (but see Bolinger, 1973, for 
evidence that even expletives have some semantic content). This class of counterexamples (and many others; see Bloom, under review) suggests that the mapping from NPs to individuals does not hold in all cases. These exceptions must somehow be learnable by children through something other than the mapping posited above.

One possibility, first advanced by Nishigauchi and Roeper (1987), is that children must initially acquire a given NP by using the syntax-semantics mapping (e.g., referential 'it'), and only after having done so, can they understand the same word or string of words in a semantically empty context (e.g., expletive 'it'). This makes the prediction that across different languages, all words and phrases that are NP expletives must also be NPs of the semantically well-behaved type (NPs that refer), because otherwise children would not be able to acquire them. There is some evidence that this is the case (Nishigauchi and Roeper 1987), and one could make the further prediction that children can only categorize a string of words within an idiom as an NP (e.g., 'the bucket' as in 'kick the bucket') if they are already capable of construing that string of words as having some referential meaning when it is outside of the idiom. A theory along these lines is discussed in Bloom (under review); for the purposes here, it should be noted that a complete account of lexical acquisition must explain how children acquire these sorts of non-referential NPs. ${ }^{2}$

\subsection{Count nouns vs. mass nouns}

If we restrict their domain to count nouns, we can collapse the whole object constraint, the taxonomic constraint, and the shape bias as follows:

Count nouns refer to kinds of whole objects (Markman and Hutchinson 1984) and children are biased to extend them on the basis of shape (Landau et al. 1988).

This generalization connects with a sizable literature that attempts to discover the semantic basis of the grammatical count/mass distinction (e.g.,

2 A different sort of puzzle concerns NPs that apparently refer to kinds, as with the subject NPs 'dogs' and 'water' in the sentences 'Dogs are friendly animals' and 'Water is good to drink'. These NPs can be construed as referring not to actual dogs or actual portions of water, but to abstract kinds - to the species DOG and the substance WATER. Under one analysis, they serve as proper names for these kinds, and thus refer to kinds in a very different way than do nouns (Carlson 1977). In any case, the acquisition and representation of these NPs constitute a further domain of study from the standpoint of syntax-semantics mappings. 
Bach 1986, Bloom 1990a, 1994a; Bloomfield 1933, Gordon 1985, 1988; Jackendoff 1991, Langacker 1987, Levy 1988, McCawley 1975, McPherson 1991, Mufwene 1984, Quine 1960, Ware 1979, Weinrich 1966, Whorf 1956, Wierzbicka 1985, see Gathercole 1986 for a review). In general, it is clear that objects tend to be described by count nouns ('a dog', 'five tables') and nonsolid substances tend to be described by mass nouns ('much water', 'less sand'). This holds for languages other than English; across different language families, names for entities like dogs and tables are always count nouns, and names for entities like water and sand are always mass nouns (Markman 1985. Mufwene 1984, Wierzbicka 1985). This pattern is unlikely to be a coincidence, and it might lead one to the hypothesis that entities described by count nouns have perceptually salient boundaries, and thus are countable, while mass nouns describe everything else.

Nevertheless, the same objections discussed earlier against the whole object constraint have also been made against this theory of count/mass syntax (e.g., by Ware 1979). One objection is that many count nouns do not describe whole objects; there exist abstract words like 'nap' and 'joke', as well as collective nouns like 'forest' and 'army'. If one is to retain the notion that the grammatical count/mass contrast maps systematically onto some cognitive division, the cognition side of the mapping must be considerably more abstract than 'whole object' and 'non-solid substance'.

As a result of these considerations, many scholars have proposed that the grammatical count/mass distinction maps onto the semantic contrast between nouns that refer to kinds of individuals vs. those that refer to kinds of nonindividuated entities, which we can view as 'portions' (see Bach 1986, Bloom 1990a, 1994a; Bloom and Keleman, under review; Gordon 1982, 1985, 1988; Jackendoff 1983, 1990, 1991; Langacker 1987, 1990; Macnamara 1986, Macnamara and Reyes 1990). The cognitive notion of inviduals is related to properties such as countability, indivisibility, and boundedness, and is roughly equivalent to 'discrete bounded entity'. Within the domain of material entities, this usually corresponds to whole objects, though it can also correspond to entities such as a forest, a puddle, and a pile. Outside of the domain of material entities, an event that takes a bounded interval of time ('a race', 'a conference') can be construed as an individual, as can a mental state ('a headache', 'a nightmare') or a period of time ('a day', 'an hour'). (Some speculations about the precise notion of 'individual' will be discussed in section 6.2.)

What support is there for the claim that count/mass syntax actually corresponds to these aspects of abstract cognition? One source of support is from linguistic analyses (e.g., Barwise and Cooper 1981, Jackendoff 1991, 
Langacker 1987). Unless one assumes that there is some consistent semantic property holding across both material count nouns and abstract count nouns, it is difficult to provide a consistent theory of quantification. In many important regards, an NP such as 'a dog' is semantically equivalent to an NP such as 'a nightmare', and one way to capture this is by describing ' $a$ ' as having the semantic role of combining with nouns that refer to kinds of individuals to form an NP that can denote a single individual. Nouns such as 'dog' and 'nightmare' - but not nouns such as 'water' and 'advice' - refer to kinds of individuals and, thus can be used with count noun syntax.

There also exists empirical evidence concerning the productive use of these syntax-semantics mappings in adults. In one study, adults were taught novel words referring to sensations or sounds (Bloom 1994a). The syntax of the word was kept neutral with regard to its count/mass status; what varied was whether the new word was described as referring to something that occurs in discrete units of time (temporal individuals) or to something occurring over continuous periods of time (temporal portions). This had the predicted effect on adult categorization of the new word: Names for temporal individuals tended to be categorized as count nouns, while names for temporal portions tended to be categorized as mass nouns.

We can now posit the following three mappings:

Mapping 1: NPs refer to individuals

Mapping 2: Count nouns refer to kinds of individuals

Mapping 3: Mass nouns refer to kinds of portions

Before turning to the question of precisely how these three mappings enable children to acquire new words - and how they fare relative to the sorts of hypotheses advanced by Markman and others - it is necessary to consider whether young children actually possess this understanding of the relationship between syntax and semantics.

\section{Syntax-semantics mappings in young children}

\subsection{Arguments against early competence}

What is the evidence regarding children's knowledge? With regard to the count/mass distinction, it is often argued that children are insensitive to the 
semantic basis of this distinction until a relatively late age. For instance, Levy (1988: 186) reviews the work of Gordon and Gathercole and concludes as follows:

'Thus, Gathercole's conclusions are in complete agreement with the conclusions reached by Gordon (1985); namely, that children first learn the linguistic distinction as a morphosyntactic rather than a semantic distinction.'

Others have reached similar conclusions. Thus Schlesinger (1988: 147), in his discussion of domains in which his semantic assimilation theory does not apply, states:

‘.. Gordon (1985) and Gathercole (1985) have shown that the count-mass distinction is acquired through formal clues rather than via the semantic object-substance distinction. The reason seems to be that, in English, there is not a very consistent correlation between these two distinctions.'

These findings have been taken as strong evidence for a 'distributional theory' of language development. Levy (1988) argues, following KarmiloffSmith (1979), that children view the acquisition of grammar as a formal puzzle, 'a problem space per se', and semantics is irrelevant. This is also Gathercole's (1985) conclusion, but Gordon $(1985,1988)$ proposes a quite different view, maintaining that count/mass syntax is based on quantificational semantics from the very start. What young children lack, according to Gordon, is knowledge of how this semantic contrast maps onto perception. That is, they understand that the contrast between count nouns and mass nouns corresponds to the distinction between words that refer to kinds of individuals vs. words that refer to kinds of portions - but they lack an understanding that physical objects are canonical individuals and non-solid substances are canonical portions. If this were correct, then these mappings would be useless as a source of constraint in word learning.

The specific studies of Gathercole and Gordon are critically reviewed in considerable detail in Bloom (1990a); it will suffice here to raise a conceptual point. All of the experiments purportedly showing that children's understanding of count/mass is not semantic involve studying children's sensitivity to linguistic cues. Thus one finding is that if 3- and 4-year-olds hear (e.g.) 'This is $a$ blicket' they tend to grammatically categorize 'blicket' as a count noun, while if they hear 'This is some blicket', they tend to grammatically categorize 'blicket' as a mass noun. Further, children will give these syntactic cues priority over referential cues. If they hear 'This is $a$ blicket' they are 
likely to interpret the word as a count noun regardless of whether they are being shown an object or a substance (Gordon 1985).

One interpretation of this result is that children's understanding of count/ mass is not semantic. Instead children possess some generalization of the form: 'Everything following the word " $a$ " is a count noun', and this is distinct from any semantic understanding, which has to be learned at some later point. This assumes a dichotomy between 'linguistic cues' and 'semantic cues', where the latter is restricted to information that children receive through perception of the external world. An alternative, however, is to reject this dichotomy altogether. Semantic information can be conveyed through language; when children hear 'a blicket' and categorize 'blicket' as a count noun, they may be drawing a semantic inference. Specifically, children might encode the determiner ' $a$ ' as having the semantic potential of interacting with a noun that refers to a kind of individual to pick out a single individual because this is what it means - and it follows from this that any noun that follows ' $a$ ' must refer to a kind of individual and thus must be a count noun.

In fact, linguistic cues are a more reliable cue to the semantic status of a novel word than perceptual cues are. This is because a given percept can be construed in different ways; if a solid object is described as 'blicket', it is quite possible that 'blicket' is actually a mass noun, because it could refer to the stuff that the object is made out of. But linguistic cues are flawless; every noun that can co-occur with a quantifier that has the semantic role of individuation has to be a count noun. Given this, the child's early sensitivity to linguistic information actually supports a semantic theory; it does not refute it.

\subsection{Arguments for early competence}

What positive evidence exists that children possess the requisite syntaxsemantics mappings? Gordon $(1982,1985,1988)$ provides a learnability argument: Children must be capable of using semantic information when acquiring the grammatical count/mass distinction, because a non-semantic distributional analysis would have to sift through several billion possibilities, and children have productive command of count/mass syntax by about the age of 2-and-a-half. More generally, the argument is that some semantic categorization would have to be done by children in order for them to so rapidly converge on the correct linguistic generalizations, because no other source of information serves to distinguish count nouns from mass nouns in the input they receive (see also Bloom 1994a). 
Children's errors provide further evidence that they are exploiting syntaxsemantics mappings. Words that are mass nouns in English but which refer to entities that can be construed as discrete objects, like 'money', 'furniture', and 'bacon', are occasionally misencoded as count nouns, e.g., young children will sometimes say things such as 'a money' (Bloom 1994a). These errors are significantly more frequent than errors with more 'canonical' mass nouns, such as 'water' and 'milk', which refer to substances. This suggests that the categorization of new words as either count or mass is facilitated (and sometimes hampered by) children's use of mappings from syntax to semantics.

A further source of support is experimental. In a classic study, Brown (1957) showed 3- to 5-year-olds sets of pictures, one that depicted an object, another that depicted a substance, and told them to either 'show me a sib' or 'show me sib'. Children tended to point differently as a function of the syntax; when given a count noun they would tend to point to the object; when given a mass noun, they would tend to point to the substance.

More recently, Soja (1992) found a sensitivity to syntax-semantics mappings as soon as children start to productively use count/mass syntax in their spontaneous speech. When these 2-year-olds are taught a mass noun that describes a pile of stuff, they tend to construe it as a name for that kind of stuff (i.e., as having a similar meaning to 'clay'), but when taught a count noun that describes a pile of stuff, many appear to construe it as referring not to the stuff itself, but to the bounded pile (i.e., as having a similar meaning to words like 'puddle' or 'pile'). Interestingly, this effect of syntax was limited to the stuff-condition: when children were taught count nouns and mass nouns describing a novel object, few of the children construed the mass noun as referring to the stuff that the object was made of (i.e., they would not construe it as having the same meaning as words like 'wood' or 'metal'). Regardless of the count/mass status of the noun, they would tend to interpret it as a name for that kind of object. An explanation for this asymmetry will be discussed in section 5 .

There is less evidence that young children can extend the semantic implications of count/mass syntax to non-material entities, but there is one relevant study (Bloom 1994a). Here, 3- and 4-year-olds were taught names for perceptually ambiguous stimuli, which could be construed as either a set of individuals or as an unindividuated portion. In one condition, the stimulus was food, either lentils or colored pieces of spaghetti, and was the sort of entity that could be easily named with either a count noun or a mass noun. In another condition, the stimulus was a string of bell sounds from a tape- 
recorder, presented one after the other at a very fast rate, which could be construed either as a set of discrete bells or as an undifferentiated noise and therefore could also be described with either a count noun or a mass noun.

All children were presented with both the 'food' and the 'bell' stimuli. One group was told: 'These are feps - there really are a lot of feps here' (count noun condition); the other group was told: 'this is fep - there really is a lot of fep here' (mass noun condition). Then the children who were taught the word as a count noun were told to 'give the puppet a fep' for the food condition and, in the sound condition, were given a stick and a bell and asked to 'make a fep'. The children who were taught the word as a mass noun were told to 'give the puppet fep' in the food condition or, in the sound condition, to 'make fep' with the stick and the bell. If children are sensitive to the semantic properties of count/mass syntax, they should act differently in the count condition than in the mass condition. When asked for 'a fep', they should tend to give one object or make one sound, and when asked for 'fep', they should tend to give a handful of objects or make a lot of sounds.

These were the results obtained: Both 3- and 4-year-olds performed significantly above chance on both the food and sound conditions. This finding provides further support for the hypothesis that there is a semantic basis to count/mass syntax even for non-material entities, and indicates that an understanding of mappings between syntax and semantics is present in 3- and 4-year-olds.

Sensitivity to the semantics of the noun/NP contrast is evident at an even earlier age than is an understanding of the semantic basis of count/mass. The mapping hypothesis is that young children should understand that the grammatical contrast between words that are nouns and words that are NPs corresponds to the contrast between words that refer to kinds and words that refer to individuals. In a classic study by Katz et al. (1974), the experimenter taught young children new words by pointing to an object and saying either 'This is a wug' (count noun context) or 'This is wug' (NP context). Even some 17 -months-olds were sensitive to this grammatical difference; when the word was presented as a noun they tended to construe the word as the name for a kind, but when it was presented as an NP, they tended to construe it as a name for a particular individual (see Gelman and Taylor, 1984, for a replication with slightly older children). The findings that children younger than two treat nouns and NPs differently with regard to how they interact with determiners and adjectives, and that they categorize pronouns and proper names as NPs (Bloom 1990b) constitute further evidence that children possess some grasp of syntax-semantics mappings. 


\section{Subsuming the constraints}

At this point, we can return to the constraints discussed in section 1 and examine the extent to which they can be replaced by the hypothesized syntaxsemantics mappings. The taxonomic constraint is subsumed by Mappings 2 and 3, which specify that both count nouns and mass nouns refer to 'kinds' and thus have generic reference in the sense discussed by Markman. Proper names and pronouns, as they fall into the grammatical class of NPs, are in the domain of Mapping 1 and thus refer to particular individuals.

The whole object constraint - that novel words refer to whole objects - can be argued to result from the interaction between Mapping 2 (which states that count nouns refer to kinds of individuals) and a non-linguistic bias to construe discrete physical objects (DPOs) as individuals (Shipley and Shepperson 1990). That is, independent of grammar, children are very strongly biased to encode objects as individuals. When shown a display of objects and asked to count kinds ('How many kinds of animals?') or properties ('How many colors?'), they will show a strong tendency to ignore the question and to only count the discrete objects (Shipley and Shepperson 1990). Note that children are quite able to count individuals that are not objects, such as sounds and actions (Wynn 1990). What the Shipley and Shepperson study shows, however, is that in the material domain, objects stand out as individuals.

This is apparently a cognitive phenomenon, having to do with the nature of the category 'individual'; it does not directly result from the syntax-semantics mappings. But when the DPO interacts with Mapping 2, it leads to a tendency to construe count nouns as names for whole objects. This relates to young children's unwillingness to initially interpret a noun, either count or mass, that refers to a novel bounded entity as naming the stuff that the entity is made of (e.g., Dickinson 1988). In other words, the DPO causes children to strongly favor interpreting a word that describes an object as having a meaning like 'desk' (and not 'wood'), even if the word is presented with mass noun syntax. Although children show some evidence for a parallel bias to construe words describing substances as kinds of portions, this is not as powerful and will not override syntactic cues to the contrary. Thus 2-yearolds are quite willing to interpret a count noun that describes a substance as referring to that kind of bounded entity (as having a meaning like 'puddle'), even though their default assumption is that a word describing a substance will name the kind of portion (as having a meaning like 'water') (Soja 1992).

The shape bias posited by Landau et al. (1988) can be recast as a claim about how children think about objects; at least for some sorts of objects, 
they tend to initially view the appropriate dimension for generalizations as being made on the basis of shape, and this is viewed as a better basis for extending the noun usage than properties such as color, size, or texture. (Recall that this does not apply for names for substances, where properties such as texture and color are more relevant; Soja et al. 1991.) The appeal to count nouns is relevant only insofar as count nouns are the only linguistic category that specifically pick out whole objects in the material domain, and thus any bias to favor shape is most likely to apply for this set of nouns. But the nature of the bias has to do with children's understanding of object kinds, not of count nouns. ${ }^{3}$ Some support for this interpretation comes from the finding that the bias towards shape appears to shift in the course of development, presumably as the result of the child's expanding understanding of how different categories of objects might be generalized in different ways (Becker and Ward 1991, Macario 1991).

Finally, consider the procedures of Soja et al. (1991). A solid object is likely to be construed as an individual, and thus a noun (but not an NP, adjective, or verb) that describes such an object is likely to be a count noun and refer to that kind of object. Similarly, a substance is likely to be construed as a portion and thus any noun that describes such a substance is likely to be a mass noun and refer to that kind of portion. Exceptional cases such as 'wood' and 'puddle' are fully consistent with Mappings 2 and 3, but, as discussed above, the mapping from mass nouns to kinds of portions may be difficult for children to exploit in cases where the portions are solid substances, as it runs afoul of the general cognitive bias to treat objects as individuals. Put differently, to learn a word like 'wood' the child must construe an object as a unit of stuff, rather than as a single individual, and this violates the bias to construe discrete physical objects as individuals.

\section{Open questions}

\subsection{Is there developmental change?}

The claim above is that 1- and 2-year-olds possess unlearned mappings from count nouns to individuals and mass nouns to non-individuated entities,

3 Landau et al. (1988) argue that children's different responses on linguistic and non-linguistic tasks (they treat shape as more relevant for the former) suggests that the shape bias is special to language. As suggested above, however, an alternative is that the use of the count noun informs the children that the task has to do with objects (where shape is very relevant); when the noun is not present the children might just as well assume that the task has to do with properties (where 
as well as the bias to construe whole objects as individuals. Is it necessary to posit this sort of abstract knowledge, or might children start off with a simpler representation, linking up the count/mass distinction directly to the contrast between bounded and unbounded physical entities, and only later developing the more abstract adult understanding? It appears that even 2year-olds possess a cognitive understanding of 'count noun' that includes bounded substances and is thus not limited to the category of 'whole object' (Soja 1992), but the question remains of whether this understanding is initially restricted to the material domain, or whether it can extend to sounds, events, collections, and so on.

In the absence of any evidence for developmental change, one might argue that lack of a child-adult difference should be viewed as the null hypothesis in psychology (Fodor 1975, Macnamara 1982) - an argument that gains force from the fact that we have as yet no understanding of how a cognitive notion can become 'more abstract'. But cases of representational change do appear to exist (see Carey 1986, 1988), and so it remains an open question whether syntax-semantics mappings are yet another domain where children differ from adults.

No decisive evidence exists at this point, but there are three sources of evidence suggesting that the abstract adult-like understanding is present in very young children.

First, some evidence concerning early possession of the notion of "individual' emerges from the research of Starkey et al. (1990), who found that 6- to 8-month-olds possess a unified concept containing both whole objects and temporally bounded sounds. In one study, infants were exposed to either two sounds or three sounds. Immediately following this, two pictures werc simultaneously shown to the infants, one with two objects and one with three objects. The subjects tended to look longer at the picture which showed the same number of objects as there were sounds, providing some evidence that infants possess notions of 'two individuals' and of 'three individuals', where 'individual' encompasses both sounds and objects. Along the same lines, Wynn (1990) discovered that almost immediately after children are able to use the linguistic counting system to count objects, they can also use it to count sounds and events. These studies suggest that, quite independently of syntax, children do have the appropriate abstract semantic notion of 'individual'.

\footnotetext{
shape may have less priority). Note incidentally that this non-linguistic construal of the shape bias appears to be more consistent with the theory of objects and places advanced in Landau and Jackendoff (1993).
} 
Second, as noted above, children appear to be capable, even at a very early age, of productively and appropriately using words that refer to non-material entities, such as temporal intervals ('day', 'minute') events ('bath', 'nap') and abstract entities ('story', 'joke'). If it turns out that (i) they encode these words as falling into the grammatical category of 'count noun' and (ii) they understand them in the same way that adults do, then this would show that the abstract understanding of the count/mass distinction is present in 2-yearolds. But there is no strong support at present for either of these claims, at least not for children younger than three.

Finally, there is the experimental study noted above (Bloom 1994a), which showed that 3- and 4-year-olds are sensitive to the application of quantificational syntax in a domain of non-material entities (sounds). Once again, however, evidence for this sort of capacity on the part of younger children does not yet exist. At this point, then, it remains possible that the semantic category 'individual' emerges from some sort of simpler representation, such as 'bounded physical entity'.

\subsection{What is the nature of ' $k$ ind of individual'?}

This brings us to the second concern. Without a substantive theory of the precise nature of 'kind of individual', the sort of account proposed here runs the risk of being empty. Despite the central role of this notion in semantic theories of quantification and reference, we have as yet little understanding of how it links up with perception and non-linguistic cognition, and how it serves to constrain the extent of possible word meanings.

If we assume that count nouns refer to kinds of individuals, it is apparent that the reference of such nouns includes bounded substances, periods of time, events, mental states, collections of objects, and abstract social constructs. There are several hypotheses about what all of these referents have in common, and thus what the core of this semantic notion is. Some suggestions include boundedness, having a single functional role, and proximity or connectedness of parts (see e.g., Hirsch 1982, Jackendoff 1991, Langacker 1987, 1990).

Consider, for instance, count nouns that name collections, such as 'forest', 'family' and 'army'. Although they describe material entities, they violate the generalization that a count noun refers to a kind of object. As such, they are problematic for theories that posit a privileged link between words (or count nouns) and kinds of whole objects. The premise of the mapping theory sketched out above is that although there exist semantic constraints on what 
can be a possible count noun, these are based on the semantic category 'kind of individual', not 'kind of object'. One could thus describe the acquisition of words such as 'forest' by assuming that a collection of trees is construable by children as being a single individual and therefore a word that refers to that kind of individual is learnable as a count noun. But what is it about forests that makes them construable by children and adults as individuals? Why do children readily construe a group of trees as a possible individual ('a forest'), and yet do not construe all of the leaves of a tree as a single individual (see Chomsky and Walker 1978)?

One tentative proposal is as follows:

Hypothesis about possible individuals:

Something (e.g., an object or set of objects) can be encoded as an 'individual' if we can construe it as playing an independent causal role in some conceptual domain.

The intuition underlying this is as follows: We view something as an individual only if doing so allows us to better understand and predict the causal relationships that hold within a given conceptual domain. The strongest example is the case of bounded objects; these are the canonical example of 'individuals' within the physical domain and are highly privileged in the course of development. Infants are predisposed to analyze their chaotic sensory input into a world of distinct bounded objects that persist over time and space (e.g. Spelke 1988). This mode of interpretation is likely to have evolved because construing the environment in these terms is the best way to make sense of what is going on, and allows for the most predictive power. Any primate that lacked this conceptual scheme would fail to respond to the world in a timely and effective manner - it would not be able to track prey and avoid predators, for example - and would not survive.

The bias to construe objects as individuals is the result of evolution, not learning, and this may hold as well for individuation within other domains, such as social cognition or naive theories of mind. For instance, humans and other primates might be predisposed to classify certain social groups as individuals for the purpose of inference and prediction; as such, notions like 'family' and 'social group' might be innate (Hirschfeld 1987, Jackendoff 1992). But for most domains, we have to discover the relevant individuals in the course of understanding the nature of the domain. One would have a difficult time understanding geography or politics, for instance, without the 
ability to understand entities like 'Canada' and 'France' as discrete individuals that causally interact with each other.

Returning to names for collections, it is clear that one's conceptual framework also determines the specific conditions under which a set of objects can be construed as forming a collection. The adult understanding of 'forest' is of a group of trees growing together in a certain environment - for the word to apply, there have to be a sufficient number of trees and they must be bunched together, but they need not form a precise shape. Nouns such as 'family' have an even looser spatial restriction: they can apply even if the elements that make up the collection bear no non-trivial spatial relationship at all; it is sensible to say 'that family is scattered around the world' (compare the semantic oddness of 'that forest is scattered around the world'.) But now consider Donald Judd's sculpture 'untitled' (1928), composed of 10 Plexiglas pieces mounted vertically on a wall, separated from one another by exactly the same distance. In this case, the precise configuration does matter; the intuition would be that two rows of five Plexiglas pieces stacked on a table would be different from 'untitled' and Judd might reasonably view this modification as a destruction of his artwork.

If the notion of 'individual' can be related to notions of intentionality and social interaction, it follows that, in principle, any set of objects can be construed as a single individual and thus named with a count noun. There is no count noun in English referring to a single shoe and a single glove (i.e., such that exactly one shoe and one glove would be 'one fizzbit') but if such a pair of objects was exactly what one needed in order to participate in some kind of religious ceremony, such a name would probably be learnable by someone trying to make sense of that ceremony. One intriguing example of this again involves artwork; a proper name (e.g., 'January Angst') might describe six concrete columns surrounded by broken glass. Although this set of objects is an individual solely by virtue of the artist's intention, this fact is sufficient for adults (and possibly children) to acquire and understand this new name.

Current research addresses these issues by exploring the circumstances under which people will give a collective interpretation for a set of discrete objects. One methodology used is to show adults a set of four identical objects and tell them either 'this is a fendle' (count syntax) or 'the new word for this is: fendle' (neutral syntax). Thus the novel word could either be a collective noun, like 'forest', and refer to all four objects, or it could be an object name, like 'tree', and refer to a single object. Then the adults are shown other displays with the same kind of objects used in the training phrase, such as a display with one object and a display with eight 
objects, and asked to describe these using the new word. Their responses indicate whether they think the word refers to a collection or to an individual object. For instance, if they interpret 'fendle' as a collective noun, they should describe eight objects as either 'one fendle' or 'two fendles' (depending on how crucial numerosity is in their collective interpretation) and should describe one object as 'part of a fendle'. If they interpret it as an object name, they should describe eight objects as 'eight fendles' and one object as 'one fendle'.

In a pilot study with a group of 36 adults, we tested the effects of syntax (singular count vs. neutral) and 'intentional integrity' on their interpretation of novel words. This second manipulation went as follows: For half of the trials, the objects were placed in front of the subject slowly and carefully; for the other half, the objects were casually dumped in front of the subject. The prediction was that this simple manipulation would have an effect on whether the subjects construe the novel word as a collective noun. The mere act of purposefully and intentionally setting out the stimuli in a given configuration should be sufficient to emphasize to the subject that the set itself is relevant as a single individual.

This prediction was confirmed: When the novel word was presented as a singular count noun, there was a bias towards interpreting it as referring to the entire collection. The bias increased (though not significantly so) when the objects were placed in front of the subject, rather than dumped. When the novel word was presented without syntactic support, however, there was an actual switch in the favored interpretation: In the dumping condition, almost all subjects construed the word as a name for a single object (like 'tree'), but for the placing condition, the collection interpretation (like 'forest') was strongly favored.

Further research will explore whether young children can also acquire a collective noun through this sort of intentional cue, and will also focus on the precise nature of adults' and children's construal of the new word. The hope is that by studying the acquisition and understanding of nominals referring to sets of discrete objects - including collective nouns and names for artwork we will gain some insight into the nature and development of the notion 'individual' and how it relates to cognition and perception.

\subsection{How can these mappings apply prior to the acquisition of overt syntax?}

The proposal here is that the constraints children use when acquiring words are the result of their understanding of the mappings from syntactic 
categories to categories of cognition. But productive command of count/mass syntax comes in at roughly the age of 2-and-a-half and children have begun to learn words over a year prior to this. To make matters worse, there exist languages which do not appear to exploit the count/mass distinction in either syntax or morphology (e.g., Mandarin Chinese), and yet children acquiring these languages have no difficulty learning words.

Also, many scholars have argued that children use properties of word meaning to determine the syntactic categories that their very first words belong to (Bloom 1990a, 1994a, under review; Grimshaw 1981, Macnamara 1982, 1986; Pinker 1984), a proposal that has been dubbed 'semantic bootstrapping'. Thus these syntax-semantics mappings might work in both directions, to facilitate both lexical and syntactic development. But if this is correct, then very young children must have the capacity to learn at least some aspects of word meaning in the absence of syntax.

Finally, we know that syntax is not essential for adults. One can learn a word like 'pencil' perfectly well without hearing it used with count syntax (e.g., without it being preceded by a quantifier like 'a' or 'many'). This proves that overt syntax cannot be essential for word learning.

Nevertheles, even where there is no specific syntactic information, the existence of the mappings still serves to narrow down the possible construals of what a word can mean. This is because any novel word must belong to a syntactic category, and as such it must fall into one of a limited set of semantic classes. For instance, there are no words that refer to chains of thematically-related entities - no natural language could have a word that refers both to dogs and to everything that dogs standardly interact with - and the reason for this could be that there is no syntactic category that encodes such a notion. More generally, there may be no constraints on word meaning per se; there might only be constraints on possible count nouns, possible mass nouns, possible intransitive verbs, and so on. But since any word has to be either a count noun or a mass noun or an intransitive verb and so on, any word is thereby constrained as to what it can mean.

For example, Mappings 2 and 3 limit possible word meanings even before children can distinguish the grammatical markings of count nouns from those of mass nouns, because once children know that the word is a noun, they know it must be either count or mass, and the mappings limit its meaning to two semantic classes - either it refers to a kind of individual or it refers to a kind of portion. Of course, the mappings are less effective at this point, since any ambiguities (cases where children cannot tell whether the adult intends to describe an object or a substance) cannot be resolved through attention to 
overt grammatical cues. This may be one explanation for the well-known finding that 3-year-olds show a greater sensitivity to the constraints than 2year-olds (e.g., Landau et al. 1988, Markman 1989, Nelson 1988) - children can use the constraints to their fullest only once they have some facility with the grammatical and morphological structure of their language.

The view that these mappings provide constraints on children's word meanings even prior to the acquisition of surface syntax may be counterintuitive, but there is some support for it. In an extended analysis of the production and comprehension abilities of children in the one-word stage, Huttenlocher and Smiley (1987: 84) state, 'Taken as a group, the object words in the single-word period form a broad semantic class which contrasts with other semantic classes emerging at the same time. That is, the pattern of usage of object words contrasts with that of words for events ..., words for persons ..., words for temporary states, greetings, and negation, and so on'. They go on to suggest that this early demarcation of words into these classes provides a semantic foundation for the later acquisition of syntactic categories. A more radical interpretation is that these children have already classified these words into the relevant grammatical classes, and all they have left to do is learn how (or if) their language expresses these classes in the grammar and morphology. (Does their language mark the contrast between count nouns and mass nouns? Is the morphology different for verbs than for adjectives?) Once they have acquired these surface expressions of the linguistic categories, children can use the mappings to further facilitate the acquisition of word meanings.

\section{Limitations of syntax-semantics mappings}

One might be tempted to view the constraints that emerge from these syntax-semantics mappings as constituting a solution to the problem of word learning. For instance, once the child knows that 'dog' is a count noun, she can infer that the word refers to a kind of individual, and this excludes innumerable other possible interpretations. If knowing the grammatical class that a word belongs to is tantamount to knowing what it means, then these mappings could effectively solve the induction problem. Unfortunately, there are two main reasons why this optimism is misguided.

First, we have been assuming throughout that the child is able to infer what the novel word is being used to describe, leaving only the question of how she encodes the meaning and appropriately extends it to novel instances. As Gleitman (1990) has argued, however, there are many cases where children 
are exposed to words in the absence of the entities that they describe. An adult, for example, might point to a bowl of cereal and say 'Do you want milk with that?' even when no milk is present. Consider also words for nonmaterial entities, like 'nap' and 'joke', where the notion of inferring what an adult is pointing to or looking at does not apply. One could also note the success of blind children at learning words (Landau and Gleitman 1985) in order to appreciate the mystery here. A complete theory must explain how children grasp the adult's intention to refer - how they somehow make the correct guess as to what adults are talking about when they use novel names (for research along these lines, see Baldwin 1991). More generally, no theory of the acquisition of words can be complete without a prior theory of how children can pick up the intended reference of language-users (Macnamara 1982).

Second, even with the aid of grammar-cognition mappings, there is still an infinity of possible meanings of the new word and children are stuck with sorting them out. The count noun 'dog' could refer to dogs, but it could also refer to dogs and pencils, to dogs until the year 2000 and then to cats, and so on. Knowing that a given word refers to a kind of individual is only a small part of the word learning puzzle; children must also determine which kind of individual the word refers to, and it is here that the induction problem runs deep, particularly given how the notion of 'kind of individual' interacts with conceptual systems such as social cognition (see section 6.2). Crucially, an explanation of how children learn words involves a theory of psychologically possible kinds (one that includes 'dogs' and 'tails', but excludes 'dogs and pencils'). In sum, while syntax-semantics mappings may be part of the solution to how children learn new words, they are not sufficient. Not only does a complete account of the acquisition of word meaning require an explanation of how people understand the intended reference of others, it also requires a theory of conceptual representation.

\section{Concluding comments}

If one were to follow the standard course in the study of word learning, and only consider the acquisition of words like 'dog' and 'cup', it would be hard to empirically distinguish the claim that children possess special word learning constraints from the alternative that they apply syntax-semantics mappings. Both theories avoid the same hard questions - how do children determine what a new word is meant to describe, and what constitutes a 
psychologically possible kind - and both can capture the same simple phenomenon; if a child hears a single object described with a word, he or she will tend to take the word as referring to that kind of object. Within this domain, the advantage of the syntax-semantics mapping theory is solely theoretical; it only posits aspects of children's psychology (a mapping from count nouns to kinds of individuals and a bias to view discrete physical objects as individuals) that have independent empirical support and have been previously proposed in adults for reasons that have nothing to do with lexical acquisition. This is preferable to having to posit special unlearned constraints that exist solely to help children acquire words and which have no other motivation or support.

The empirical differences between the two theories become more obvious when we consider words that do not describe whole objects. The constraints advanced by Markman (1990), Soja et al. (1991), and Landau et al. (1988) do not apply to words like 'Fred', 'she', 'map', 'foot', and 'forest' - and these sorts of words are present in the speech of 1- and 2-year-old children. By shifting the focus to mappings between grammar and abstract cognition, we have a framework in which to deal with the acquisition of pronouns and proper names, words for substances, words for material entities that are not whole objects (like parts and collections), and words for abstract entities.

With the notable exception of research on the development of verb meaning (e.g., Gleitman 1990, Pinker 1989), most scholars have viewed word learning as an independent issue from the nature and development of grammatical knowledge. It is also often assumed that the theoretically interesting cases of word learning are limited to the acquisition of words for material entities, usually names for whole objects. This article has presented reasons for abandoning both of these assumptions, and for exploring how mappings between syntactic categories like 'count noun' and abstract semantic categories like 'kind of individual' facilitate the acquisition of word meaning.

\section{References}

Au, T.K. and M. Glusman, 1990. The principle of mutual exclusivity in word learning: To honor or not to honor? Child Development 61, 1474-1490.

Au, T.K. and E.M. Markman, 1987. Acquiring word meanings via linguistic contrast. Cognitive Development 2, 217-236.

Bach, E., 1986. The algebra of events. Linguistics and Philosophy 9, 5-16.

Baldwin, D.A., 1989. Priorities in children's expectations about object label reference: Form over color. Child Development 60, 1291-1306. 
Baldwin, D.A., 1991. Infants' contribution to the achievement of joint reference. Child Development 62, 875-890.

Barwise, J. and R. Cooper, 1981. Generalized quantifiers and natural language. Linguistics and Philosophy 4, 159-219.

Becker, A.H. and T.B. Ward, 1991. Children's use of shape in extending new labels to animate objects: Identity versus postural change. Cognitive Development 6, 3-16.

Benedict, H., 1979. Early lexical development: Comprehension and production. Journal of Child Language 6, 183-200.

Bloom, P., 1990a. Semantic structure and language development. Unpublished doctoral dissertation, MIT.

Bloom, P., 1990b. Syntactic distinctions in child language. Journal of Child Language 17, 343355.

Bloom, P., 1994a. Semantic competence as an explanation for some transitions in language development. In: Y. Levy (ed.) Other children, other languages: Theoretical issues in language development, 4l-75. Hillsdale, NJ: Erlbaum.

Bloom, P., 1994b. Recent controversies in the study of language acquisition. In: M.A. Gernsbacher (ed.), Handbook of psycholinguistics. San Diego, CA: Academic Press.

Bloom, $\mathrm{P}$. , under review. Meaning-form mappings and their role in the acquisition of nominals. Language.

Bloom, P. and D. Keleman, under review. Syntactic cues and the acquisition of collective nouns. Cognition.

Bloomfield, L., 1993. Language. New York: Holt.

Bolinger, D., 1973. Ambient it is meaningful too. Journal of Linguistics 9, 261-270.

Brown, R., 1957. Linguistic determinism and the part of speech. Journal of Abnormal and Social Psychology 55, 1-5.

Callanan, M.A. and E.M. Markman, 1982. Principles of organization in young children's natural language hierarchies. Child Development 53, 1093-1101.

Carey, S., 1978. The child as word learner. In: M. Hallc, J. Brcsnan, A. Miller (eds.), Linguistic theory and psychological reality, 264-293. Cambridge, MA: MIT Press.

Carey, S., 1982. Semantic development: The state of the art. In: E. Wanner, L.R. Gleitman (eds.), Language acquisition: The state of the art, 347-389. New York: Cambridge University Press.

Carey, S., 1986. Conceptual change in childhood. Cambridge, MA: MIT Press.

Carey, S., 1988. Conceptual differences between children and adults. Mind and Language 3, 167181.

Carlson., G., 1977. Reference to kinds in English. Unpublished doctoral dissertation, Department of Linguistics. University of Massachusetts, Amherst.

Chomsky, N., 1981. Lectures on government and hinding. Foris: Dordrecht.

Chomsky, N. and E. Walker, 1978. The linguistic and psycholinguistic background. In: E. Walker (ed.), Explorations in the biology of language, 15-26. Cambridge, MA: MIT Press.

Clark, E.V., 1973. What's in a word? On the child's acquisition of semantics in his first language. In: T.E. Moore (ed.), Cognitive development and the acquisition of language, 65-110. New York: Academic.

Clark, E.V., 1987. The principle of contrast: A constraint on language acquisition. In: B. MacWhinney (ed.), Mechanisms of language acquisition, 1-33. Hillsdale, NJ: Erlbaum.

Clark, E.V., 1990. On the pragmatics of contrast. Journal of Child Language 17, 417-432.

Di Sciullo, A. and E. Williams, 1987. On the definition of word. Cambridge, MA: MIT Press. 
Dickinson, D.K., 1988. Learning names for materials: Factors constraining and limiting hypotheses about word meaning. Cognitive Development 3, 15-35.

Fodor, J., 1975. The language of thought. New York: Crowell.

Gathercole, V.C., 1985. 'He has too many hard questions': The acquisition of the linguistic masscount distinction in much and many. Journal of Child Language 12, 395-415.

Gathercole, V.C., 1986. Evaluating competing theories with child language data: The case of the count-mass distinction. Linguistics and Philosophy 6, 151-190.

Gathercole, V.C., 1987. The contrastive hypothesis for the acquisition of word meaning: A reconsideration of the theory. Journal of Child Language 14, 493-531.

Gelman, S.A. and M. Taylor, 1984. How two-year-old children interpret proper and common names for unfamiliar objects. Child Development 55, 1535-1540.

Gentner, D, 1982. Why nouns are learned before verbs: Linguistic relativity versus natural partitioning. In: S.A. Kuczaj (ed.), Language development, Vol. II: Language, thought, and culture, 301-334. Hillsdale, NJ: Erlbaum.

Gleitman, L.R., 1990. The structural sources of word meaning. Language Acquisition 1, 3-55.

Goodman, N., 1983. Fact, fiction, and forecast. Cambridge, MA: Harvard University Press.

Gopnik, A. and S. Choi, 1990. Do linguistic differences lead to cognitive differences? A crosslinguistic study of semantic and cognitive development. First Language 10, 199-215.

Gordon, P., 1982. The acquisition of syntactic categories: The case of the count/mass distinction. Unpublished doctoral dissertation, MIT.

Gordon, P., 1985. Evaluating the semantic categories hypothesis: The case of the count/mass distinction. Cognition 20, 209-242.

Gordon, P., 1988. Count/mass category acquisition: Distributional distinctions in children's speech. Journal of Child Language 15, 109-128.

Grimshaw, J., 1981. Form, function, and the language acquisition device. In: C.L. Baker, J. McCarthy (eds.), The logical problem of language acquisition, 183-210. Cambridge, MA: MIT Press.

Ilirsch, E., 1982. The concept of identity. New York, NY: Oxford University Press.

Huttenlocher, J. and P. Smiley, 1987. Early word meanings: The case of object names. Cognitive Psychology 19, 63-89.

Jackendoff, R., 1983. Semantics and cognition. Cambridge, MA: MIT Press.

Jackendoff, R., 1990. Semantic structures. Cambridge, MA: MIT Press.

Jackendoff, R., 1991. Parts and boundaries. Cognition 41, 9-45.

Jackendoff, R., 1992. Is there a faculty of social cognition? In: R. Jackendoff (ed.), Languages of the mind: Essays on mental representation, 69-81. Cambridge, MA: MIT Press.

Karmiloff-Smith, A., 1979. A functional approach to language acquisition. New York: Cambridge University Press.

Katz, N. E. Baker and J. Macnamara, 1974. What's in a name? A study of how children learn common and proper names. Child Development 45, 469-473.

Keil, F.C., 1989. Concepts, kinds, and cognitive development. Cambridge, MA: MIT Press.

Lakoff, G., 1987. Women, fire, and dangerous things: What categories reveal about the mind. Chicago, IL: Chicago University Press.

Landau, B. and L.R. Gleitman, 1985. Language and experience. Cambridge, MA: Harvard University Press.

Landau, B. and R. Jackendoff, 1993. 'What' and 'where' in spatial language and spatial cognition. Behavioral and Brain Sciences 16, 217-238. 
Landau, B, S. Jones and L. B. Smith, 1992. Perception, ontology, and naming in young children: Commentary on Soja, Carey, and Spelke. Cognition 43, 85-91.

Landau, B., L. B. Smith and S. Jones, 1988. The importance of shape in early lexical learning. Cognitive Development 3, 299-321.

Langacker, R.W., 1987. Nouns and verbs. Language 63, 53-94.

Langacker, R.W., 1990. Foundation of cognitive grammar, Volume II: Descriptive application. Unpublished manuscript, UCSD.

Levy, Y., 1988. On the early learning of grammatical systems: Evidence from studies of the acquisition of gender and countability. Journal of Child Language 15, 179-186.

Macario, J., 1991. Young children's use of color in classification: Foods and canonically colored objects. Cognitive Development 6, 17-46.

Macnamara, J., 1982. Names for things: A study of human learning. Cambridge, MA: MIT Press.

Macnamara, J., 1986. A border dispute: The place of logic in psychology. Cambridge, MA: MIT Press.

Macnamara, J. and G. Reyes, 1990. The learning of proper names and count nouns: Foundational and empirical issues. Unpublished manuscript.

Markman, E.M., 1985. Why superordinate categories can be mass nouns. Cognition 19, 31-53.

Markman, E.M., 1989. Categorization and naming in children: Problems of induction. Cambridge, MA: MIT Press.

Markman, E.M., 1990. Constraints children place on word meanings. Cognitive Science 14, $57-$ 77.

Markman, E.M. and J.E. Hutchinson, 1984. Children's sensitivity to constraints in word meaning: Taxonomic versus thematic relations. Cognitive Psychology 16, 1-27.

Markman, E.M. and G.F. Wachtel, 1988. Children's use of mutual exclusivity to constrain the meaning of words. Cognitive Psychology 20, 121-157.

McCawley, J., 1975. Lexicography and the count-mass distinction. Proceedings of the first annual conference of the Berkeley Linguistics Society.

McPherson, L., 1991. 'A little' goes a long way: Evidence for a perceptual basis of learning for the noun categories COUNT and MASS. Journal of Child Language 18, 315-338.

Mervis, C.B., R.M. Golinkoff and J. Bertrand, 1991. Young children learn synonyms: A refutation of mutual exclusivity. Poster presented at the Biennial Meeting for the Society for Research in Child Development, Seattle, WA, April, 1991.

Mufwene, S., 1984. The count/mass distinction and the English lexicon. 1984 CLS Parasession on Lexical Semantics.

Naigles, L., 1990. Children use syntax to learn verb meanings. Journal of Child Language 17, 357-374.

Nelson, K., 1988. Constraints on word meaning? Cognitive Development 3, 221-246.

Nelson, K., 1990. Comment on Behrend's 'Constraints and development'. Cognitive Development 5, 331-339.

Nelson, K., J. Hampson and L.L. Shaw, 1993. Nouns in early lexicons: Evidence, explanations, and extensions. Journal of Child Language 20, 61-84.

Nishigauchi, T. and T. Roeper, 1987. Deductive parameters and the growth of empty categories. In: T. Roeper, E. Williams (eds.), Parameter-setting and language acquisition, 91-121. Dordrecht: Reidel.

Parsons, T., 1990. Events in the semantics of English. Cambridge, MA: MIT Press. 
Pinker, S., 1984. Language learnability and language development. Cambridge, MA: Harvard University Press.

Pinker, S., 1989. Learnability and cognition. Cambridge, MA: MIT Press.

Prasada, S., 1993. Learning names for solid substances: Quantifying solid entities as portions. Cognitive Development 8, 83-104.

Premack, D., 1990. Words: What are they, and do animals have them? Cognition 37, 197-212.

Quine, W.V.O., 1960. Word and object. Cambridge, MA: MIT Press.

Sapir, E., 1921. Language. New York: Harcourt, Brace.

Schlesinger, I.M., 1988. The origin of relational categories. In: Y, Levy, I. M. Schlesinger, M.D.S. Braine (eds.), Categories of processes in language acquisition, 121-178. Hillsdale, NJ: Erlbaum.

Shipley, E.F. and B. Shepperson, 1990. Countable entities: Developmental changes. Cognition $34,109-136$.

Soja, N.N., 1987. Ontological constraints on 2-year-olds' induction of word meanings. Unpublished doctoral dissertation, MIT.

Soja, N. N., 1992. Inferences about the meanings of nouns: The relationship between perception and syntax. Cognitive Development 7, 29-45.

Soja, N.N., S. Carey and E.S. Spelke, 1991. Ontological categories guide young children's inductions of word meaning: Object terms and substance terms. Cognition 38, 179-211.

Soja, N. N., S. Carey and E.S. Spelke, 1992. Perception, ontology, and word meaning. Cognition 45, 101-107.

Spelke, E.S., 1988. Where perception ends and thinking begins: The apprehension of objects in infancy. In: A. Yonas (ed.), Minnesota Symposia on Child Psychology, 197-234. Hillsdale, NJ : Erlbaum.

Starkey, P., E.S. Spelke and R. Gelman, 1990. Numerical abstraction by human infants. Cognition 36, 97-127.

Taylor, M. and S. Gelman, 1988. Adjectives and nouns: Children's strategies for learning new words. Child Development 59, 411-419.

Ware, R., 1979. Some bits and pieces. In: F. Pelletier (ed.), Mass terms: Some philosophical problems, 15-29. Dordrecht: Reidel.

Waxman. S., 1990. Linguistic biases and the establishment of conceptual hierarchies: Evidence from preschool children. Cognitive Development 5, 123-150.

Waxman, S. and R. Gelman, 1986. Preschoolers' use of superordinate relations in classifications and language. Cognitive Development 1, 139-156.

Weinreich, U., 1966. Explorations in semantic theory. In: T. Sebeok (ed.), Current trends in linguistics, Vol 3: 395-477. Mouton: The Hague.

Whorf, B., 1956. Language, thought, and reality. Cambridge, MA: MIT Press.

Wierzbicka, A., 1985. Oats and wheat: The fallacy of arbitrariness. In: J. Haiman (ed.), Iconicity in syntax, 311-342. Amsterdam: Benjamins.

Wynn, K., 1990. Children's understanding of counting. Cognition 36, 155-193. 